Metastatic prostate cancer, which shows progression despite castration testosterone levels, was previously defined as hormone-refractory. This definition has recently been changed to the one presently used - castrate-resistant prostate cancer. Numerous fundamental studies have provided evidence that the development of hormone-refractory prostate cancer is constantly dependent on the concentration of androgens. The aim of the metastatic castrate-resistant prostate cancer (mCRPC) treatment is currently to obtain the lowest possible androgen concentration. The effectiveness of such management has been proven by the results of clinical studies on the latest hormonal and chemotherapeutic medications. In the last two decades, new effective chemotherapeutics have become available on the market: abiraterone, enzalutamide, docetaxel, cabazitaxel, zoldronic acid, denosumab and alpharadin They significantly contribute to extending patients' survival and to improving their quality of life. Therefore, the question arises whether using luteinizing hormone-releasing hormone (LHRH) analogues is still a necessary element of the therapy. A detailed analysis of study regimens involving the above-mentioned medications and of available publications supports the view that LHRH analogues are the basic strategy in the treatment of patients with $\mathrm{mCRPC}$. All clinical trials evaluating new therapies still followed the principle of obtaining castration testosterone levels as a result of using LHRH analogues simultaneously with the new medications.

Key words: metastatic castrate-resistant prostate cancer, LHRH analogues, hormone therapy, chemotherapy.

Contemp Oncol (Pozn) 2014; 18 (2): 85-89 DOI: $10.5114 /$ wo.2014.42723

\section{The use of luteinizing hormone- releasing hormone analogues is still an indispensable element of therapy in castrate-resistant prostate cancer}

\author{
Tomasz Milecki ${ }^{1}$, Andrzej Antczak ${ }^{1}$, Zbigniew Kwias ${ }^{1}$, Piotr Milecki²,3 \\ ${ }^{1}$ Department and Clinic of Urology and Urologic Oncology, Poznan University of Medical \\ Science, Poland \\ ${ }^{2}$ Department of Radiotherapy, Greater Poland Cancer Centre, Poznan, Poland \\ ${ }^{3}$ Chair and Department of Electroradiology, Poznan University of Medical Sciences, \\ Poland
}

For decades, hormone-refractory prostate cancer was defined as a neoplasm, the development of which no longer depended on hormonal therapy, and thus on the testosterone concentration. In the light of recent fundamental and clinical research results, the definition of hormone-refractory prostatic cancer, i.e. cancer which progresses after the primary hormone treatment, has been revised, and has been changed to castrate-resistant prostate cancer.

One of the standards in the treatment of metastatic castrate-resistant prostate cancer (mCRPC) is hormone therapy, which aims to eliminate androgens from the blood via suppression of the hypothalamic-pituitary-gonadal axis and/or inhibition of androgen receptors by testosterone-competitive and dihydrotestosterone-competitive agents [1]. Unfortunately, hormone therapy is usually effective for a relatively short period of time, after which progression occurs as a result of resistance to the treatment.

Testosterone "fuels" cancer prostate cells, stimulating their proliferation. The androgen-dependent nature of prostate cancer was discovered in 1941 by Huggins and Hodges [2]. 95\% of testosterone is produced in Leydig cells in man's testicles [3]. Within neoplastic cells, testosterone is transformed into a more active form, dihydrotestosterone. Both compounds demonstrate affinity to the androgen receptor in the cytoplasm, and together create a complex which penetrates into the cell nucleus and binds with certain DNA sequences responsible for growth, proliferation and metabolism of neoplastic cells. To date, it has been assumed that being refractory to hormone therapy is caused by a certain vaguely defined "resistance" to hormonal treatment. However, numerous studies in which concentrations of testosterone and its derivatives were measured in the hormone-refractory cancer tissue provided evidence that castration therapy does not entirely eliminate androgens from the tumour cell environment, despite castration levels of testosterone in peripheral blood [4]. This is due to the fact that the metabolism of adrenal androgens and intracellular steroidogenesis pathways in the cancer cells responsible for de novo synthesis of androgens is still active $[5,6]$. Another important factor in the pathogenesis of castrate-resistant cancer is the increased sensitivity of neoplastic cells to very low testosterone concentrations. This is caused by over-expression of the androgen receptor, and by its mutations [7]. It has been demonstrated that even trace amounts of androgens enable activation of the mutated and multiplied androgen receptor [8]. The discovery of these relationships in the cells of cancer resistant to hormone therapy led to the hypothesis that, although previously 
treated as hormone-refractory, prostate cancer is still dependent on androgen concentrations. As a result, the commonly accepted term "castrate-resistant prostate cancer" has been introduced. The main goal of castrate-resistant cancer treatment is inhibition of the androgen-receptor axis. This effect may be achieved by a maximum reduction of androgen activity. Therefore, the basic treatment strategy is the maximum inhibition of testosterone production in the testicles as a result of using luteinizing hormone-releasing hormone (LHRH) analogues. Simultaneously, new medications are introduced, which may demonstrate a direct cytotoxic effect (docetaxel, cabazitaxel), or which may lead to a further reduction of the testosterone concentration in the external environment of the prostate cancer cell by blocking testosterone production (abiraterone). Another method of reducing the effect of androgens on a cancer cell is to use chemotherapeutic agents more effective than bicalutamide for androgen receptor inhibition, then to block the androgen receptor complex inside the cell, and finally inhibit DNA activation (enzalutamide). Despite using the mentioned medications, it should be taken into consideration that the hypothalamic-pituitary-gonadal axis is active, and it is the main source of testosterone; therefore, regardless of the failure of the first-line hormone therapy, using LHRH is still crucial for effective treatment. The validity of this assumption has not been demonstrated in randomised studies. However, retrospective studies evaluating the effects of continued hormone therapy in patients with hormone-refractory prostate cancer demonstrate longer overall survival in patients using LHRH analogues, regardless of resistance to hormones $[9,10]$. Moreover, there are no clinical studies on the use of new medications without concurrent castration therapy with LHRH analogues. Therefore, the continuation of castration therapy is a standard treatment in $\mathrm{mCRPC}$. The present consensus on effective hormonal treatment assumes obtaining blood testosterone concentration of less than $50 \mathrm{ng} / \mathrm{ml}$. However, clinical study results confirming the influence of this factor on disease progression gave rise to a discussion on the optimal cut-off value for testosterone. The present blood testosterone concentration of less than 50 $\mathrm{ng} / \mathrm{ml}$ is being questioned as too high, and a lower cut-off point of $20 \mathrm{ng} / \mathrm{ml}$ has been suggested. A retrospective analysis evaluating the effectiveness of the LHRH analogue triptorelin, administered to patients with mCRPC every 3 months, has demonstrated that this form of treatment enables a lower testosterone castration level $(<20 \mathrm{ng} / \mathrm{ml})$ to be achieved in $95 \%$ of patients after 6 months of therapy [11]. Another study, conducted by Perachino et al., assessed the effect of testosterone concentration on cancer-specific survival (CCS) in patients with $\mathrm{mCRPC}$. The study involved 129 patients who had no history of previous hormone therapy [12]. The median baseline testosterone was $410 \mathrm{ng} / \mathrm{dl}$ at the beginning of the study. The patients then underwent hormone therapy (LHRH analogue). The median testosterone nadir during the treatment was $21 \mathrm{ng} / \mathrm{dl}$, and after 6 months the median testosterone was $40 \mathrm{ng} / \mathrm{dl}$. The study demonstrated that higher testosterone concentration correlated with a higher risk of death due to prostate cancer: HR 1.333 (95\% Cl: 1.053-1.687) $p<0.050$. Based on these data, it was concluded that the aim of hormone therapy in patients with metastatic prostate cancer should be a maximum reduction of the testosterone level. As previously mentioned, the continuation of hormonal therapy with an LHRH analogue in patients with hormone-refractory prostate cancer is supported by phase III studies, involving both new generation chemotherapeutic medications used after an unsuccessful first-line therapy, and the newest hormonal medications. All clinical trials evaluating new therapies still followed the principle of obtaining castration testosterone levels as a result of using LHRH analogues, simultaneously with the new medications.

One of the first medications used in cases of progression of the prostate cancer to the metastatic castrate-resistant stage is docetaxel. Effectiveness of this chemotherapeutic has been demonstrated in two phase III clinical trials - TAX 327 and SWOG $9916[13,14]$. It is worth emphasising that participation in these studies was conditional upon continuation of the hormone treatment, whose aim was to achieve a testosterone concentration of less than $50 \mathrm{ng} / \mathrm{ml}$. The first of the studies compared the effectiveness of docetaxel with mitoxantrone. It was a three-arm study - docetaxel therapy was applied in two arms: $75 \mathrm{mg} / \mathrm{m}^{2}$ every 3 weeks or $30 \mathrm{mg} / \mathrm{m}^{2}$ once a week, and the control arm involved treatment with mitoxantrone $12 \mathrm{mg} / \mathrm{m}^{2}$. The study demonstrated that median survival in patients treated with docetaxel at $75 \mathrm{mg} / \mathrm{m}^{2}$ every 3 weeks was 19.2 months, and with mitoxantrone 16.3 months, whereas the therapy with docetaxel at $30 \mathrm{mg} / \mathrm{m}^{2}$ did not significantly affect the length of survival. The SWOG 9916 study also confirmed the effectiveness of docetaxel therapy in a slightly different configuration.

Cabazitaxel is another new generation chemotherapeutic agent, whose cytostatic effect consists in the inhibition of cell division by blocking the microtubules of the karyokinetic spindle. The TROPIC trial, assessing the effectiveness of the medication, involved patients after the firstline hormone therapy who experienced prostate cancer progression in the course of the docetaxel treatment [15]. The patients were randomised to two groups: one treated with cabazitaxel and prednisone, and the other treated with mitoxantrone and prednisone. The median overall survival for patients treated with cabazitaxel was 15.1 months vs. 12.7 months for patients receiving mitoxantrone (HR 0.70; $p<0.0001)$. Also in this case, all the patients were hormonally treated in order to achieve castration testosterone levels.

The ability to effectively block enzymes crucial for androgen synthesis has created new possibilities regarding hormone therapy. In a phase III randomised trial conducted in 2011, de Bono et al. compared the effectiveness of abiraterone combined with prednisone vs. placebo + prednisone in a second-line therapy, after unsuccessful chemotherapy with docetaxel in patients with castrate-resistant prostate cancer [16]. To achieve the lowest possible androgen concentration, the study involved patients who continued castration therapy with LHRH analogues, which was qualified as a combined therapy. The overall survival in the abiraterone group was 4 months longer than in the group without this therapy (14.8 months vs. 10.9 months).

In 2013, the results of another study with the use of abiraterone were published. The study involved over 1000 patients with castrate-resistant prostate cancer, who had no history of previous docetaxel therapy [17]. Also in this study, the therapy with LHRH analogue was continued 
concurrently with the new medication (in the absence of surgical castration). The trial demonstrated that using abiraterone significantly prolonged the radiological progression-free survival compared to the placebo group (16.5 vs. 8.3 months), and considerably extended the prostate-specific antigen (PSA) progression-free survival. Median overall survival was not achieved for the abiraterone group; however, a $25 \%$ reduction in the risk of death was demonstrated compared to the placebo group, which clearly indicates that using abiraterone prolongs the overall survival.

As abiraterone inhibits the conversion of androgens from all three sources, i.e. testicles, adrenal glands and de novo in the neoplastic cells, the question arises whether the concurrent use of an LHRH analogue (which inhibits testosterone synthesis only in the testicles) is necessary. Presently, there are no clinical studies assessing the effectiveness of abiraterone in monotherapy if the testosterone concentration is higher than $50 \mathrm{ng} / \mathrm{ml}$, and therefore, the combined treatment with abiraterone + surgical/pharmacological castration is the most effective form of androgen suppression. Another argument supporting the necessity of $\mathrm{LH}$ suppression is the presence of $\mathrm{LH}$ receptors in prostatic neoplastic cells. Pinski et al. demonstrated that stimulation of these receptors leads to increased activity of the steroidogenesis pathways enzymes, which results in an increased intracellular androgen concentration [18]. Referring to two other phase III randomised trials, it has been proven that adding androgen synthesis inhibitors to a standard LHRH analogue based hormone therapy enables a more effective reduction of androgen concentration. The combination of dutasteride and ketoconazole with total androgen blockade (TAB) in neoadjuvant therapy, 3 months prior to a radical prostatectomy in patients with locally advanced cancer, enabled the blood testosterone concentration to be reduced to $0.03 \mathrm{ng} / \mathrm{ml}$ compared with the control group $(0.92 \mathrm{ng} / \mathrm{ml})$, where the neoadjuvant therapy was limited exclusively to TAB [19]. An analogous study assessed the effect of combining abiraterone with LHRH agonists in 3 to 6 months of neoadjuvant therapy preceding a surgical treatment, and a reduction in DHT (dihydrotestosterone) from $1.3 \mathrm{ng} / \mathrm{ml}$ to $0.18 \mathrm{ng} / \mathrm{ml}$ was also achieved, as well as a reduction in DEHA and DHT concentrations in the prostate gland [20].

Enzalutamide is another new generation medication used in the treatment of MCRPC after unsuccessful chemotherapy with docetaxel, and it was approved by the FDA in 2012. Enzalutamide blocks the intracellular androgen receptor signalling pathway in three ways: through an irreversible androgen receptor antagonism (five times stronger than the effect of bicalutamide), by inhibiting translocation of androgen receptor to the nucleus, and by preventing this receptor from binding with DNA and/ or protein co-activators. In the published results of the AFFIRM trial, the effectiveness of enzalutamide at $160 \mathrm{mg} /$ day was compared with placebo in patients after unsuccessful docetaxel treatment [21]. The study involved 1199 patients who continued hormone therapy with an $\mathrm{LHRH}$ analogue. The patients were randomised at a $2: 1$ ratio enzalutamide (800 patients)/placebo (399 patients). The main endpoint of the study was overall survival. The tri- al demonstrated that enzalutamide therapy prolongs the patients' overall survival (18.4 months vs. 13.6 months in the placebo group). Other endpoints were also assessed, which also confirmed the benefits of enzalutamide treatment. It was demonstrated that enzalutamide therapy is associated with a reduction in PSA concentration by $\geq 50 \%$ ( $54 \%$ vs. $2 \% ; p<0.001)$, soft tissue response $(29 \%$ vs. $4 \% ; p<0.001)$, enhancement of the quality of life ( $43 \%$ vs. $18 \% ; p<0.001$ ), PSA progression-free survival (8.3 vs. 3.0 months; HR $0.25 ; p<0.001$ ), radiographic progression-free survival (8.3 vs. 2.9 months; HR 0.40; $p<0.001$ ), and bone-metastasis-free survival (16.7 vs. 13.3 months; HR 0.69; $p<0.001$ ). Enzalutamide was also studied within a group of patients with no history of docetaxel treatment - during the phase III PREVAIL trial [22]. Initial results of this study indicate that enzalutamide improves the overall survival and reduces by $81 \%$ the risk of radiological events compared to the placebo.

A novel retrospective study of enzalutamide for the treatment of MCRPC patient who progressed after docetaxel and abiraterone therapy has been performed, enrolling a total of 61 patients [23]. Enzalutamide resulted in a PSA decline of more than $50 \%$ in 13 (21\%) men, median progression-free survival was 12.0 weeks, the median time to PSA progression was 17.4 weeks and the median overall survival was 31.6 weeks. These data showed that some patients can still be sensitive to hormonal therapy and testosterone level even after docetaxel and abiraterone failed treatment, which is another argument why LHRH analogue therapy is still crucial for mCRPC patients.

Use of abiraterone is associated with the reduction of androgen concentrations. As a result, the neoplastic cells activate, using a feedback process, mechanisms increasing the number of androgenic receptors, thus compensating for androgenic deficiencies in the cancer cell environment, which leads to resistance. By analogy, also through a feedback mechanism, the neoplastic cells compensate the enzalutamide therapy by increasing the synthesis of androgens.

Most patients (90\%) with mCRPC develop bone metastases in the natural course of the disease, which significantly affect the quality of life and doubtlessly increase the risk of death [23-25]. The symptoms and complications associated with bone metastases are referred to in the literature as skeletal-related events (SRE), and they include pathological fractures, spinal cord compression, bone pain and palliative radiotherapy of painful bone lesions. The medications recommended in patients with bone system metastases include denosumab, zoledronic acid and alpharadin 223. However, it should be emphasised that these medications are only complementary to the LHRH analogue therapy, and they cannot be used in monotherapy. Bisphosphonates were the first group of drugs used to prevent SRE. They reveal high affinity to calcium, and, absorbed by hydroxyapatite, they are built into the bone structure, thus inhibiting its resorption. The only bisphosphonate authorised by the FDA to be used in $\mathrm{MCRPC}$ is zoledronic acid. Denosumab is a human monoclonal antibody, which binds with the RANKL ligand, thus inhibiting maturation of osteoclasts, and contributing to reduction of bone resorption. In 2011, the results of a phase III 
randomised trial comparing denosumab with zoledronic acid were published [26]. The study provided evidence that denosumab prolongs the time before the first SRE to 20.7 months, compared to 17.1 months in the patients who received zoledronic acid (HR 0.82, 95\% Cl: 0.71-0.95; $p=0.008$ ). It was also demonstrated that denosumab reduced the risk of another bone complication by $18 \%$.

Alpharadin is a new generation drug approved by the FDA in 2013, and its mode of action consists in emitting shortrange alpha radiation (ca. a range of $2-10$ cells) within the areas of high growth in the bones surrounding the metastases. The condition for the participation in the trial was the confirmation of at least two metastatic sites in the bones. The study demonstrated an increase in the overall survival of the patients with $\mathrm{MCRPC}$ who received alpharadin therapy, in comparison to the placebo (14.9 months vs. 11.3 months) [27]. Moreover, the study revealed a significant reduction in bone complications and prolonged time to their occurrence (15.6 vs. 9.8 months). Alpharadin is the first medication from the group of radiation emitters to demonstrate a beneficial effect on overall survival, simultaneously causing fewer complications than the former generation drugs. The effectiveness of denosumab, zoledronic acid and alpharadin has been confirmed in clinical trials, and they are recommended as supportive treatment in targeted therapy of bone metastases in patients with mCRPC who continue hormone therapy.

Angiogenesis is known to play a crucial role in the progression of prostate cancer. VEGF is an important factor responsible for formation of new tumour vessels [28]. One method which can prevent neovascularization is inhibition of the VEGF signalling pathway. Two new agents - bevacizumab (a humanized monoclonal antibody which inhibits major isoforms of VEGF) and aflibercept (a VEGF fusion protein which inhibits the VEGF binding receptor) - were evaluated in the phase III randomised trials VENICE (aflibercept) and CALGB
90401 (bevacizumab) $[29,30]$. The main aim of these studies was to show the prolongation of overall survival. In CALGB 90401 Kelly and colleagues compared docetaxel/prednisone and placebo with docetaxel/prednisone and bevacizumab in 1,050 men with mCRPC. The median overall survival was not prolonged significantly (22.6 months vs. 21.5 months; HR 0.91, $p=0.18$ ). Also aflibercept in combination with docetaxel/ prednisone given as first line chemotherapy for mCRPC did not lead to a statistically significant improvement in overall survival (22.1 months vs 21.2 months HR 0.94, $p=0.38$ ). In both trials the eligibility criteria included concurrent castration therapy with LHRH analogues. These two studies further underline the importance of continuation of hormonal therapy for mCRPC patients.

Tasquinimod is a novel oral drug for MCRPC patients, which has both immunomodulatory and VEGF-independent antiangiogenic properties. In a double blinded, randomized phase II trial 201 patients were enrolled [31]. Tasquinimod therapy resulted in a prolonged overall survival of 33.4 months versus placebo 30.4 months (HR 0.87, $p=0.49$ ). The best advantage was in a subgroup of 134 patients with bone metastases where patients treated with tasquinimod experienced 34.2 months overall survival compared to 27.1 months in placebo patients (HR 0.73, $p=0.19$ ). Also in this trial, patients were hormonally treated in order to achieve castration testosterone levels (Table 1).

Recently, many new medications have become available which contribute to prolonged survival and enhanced quality of life in mCRPC. They have also changed the approach to the aetiopathogenesis of the disease in advanced stages, which has led to a change in the definition: from hormone-refractory cancer to castrate-resistant cancer a stage of the disease whose development is still dependent on the presence of androgens. It should also be noted that despite the authorisation of the new medications by

Table 1. Phase III clinical trials

\begin{tabular}{|c|c|c|c|c|}
\hline Trial & Medication tested & Group of patients & No. of patients & Survival in months \\
\hline TAX 327 & $\begin{array}{c}\text { docetaxel + prednisone } \\
\text { vs. } \\
\text { mitoxantrone + prednisone }\end{array}$ & mCRPC & 1006 & 19.2 vs. 16.3 \\
\hline SWOG 9916 & $\begin{array}{c}\text { docetaxel + prednisone } \\
\text { vs. } \\
\text { mitoxantrone + prednisone }\end{array}$ & mCRPC & 674 & 17.5 vs. 15.6 \\
\hline TROPIC & $\begin{array}{c}\text { cabazitaxel + prednisone } \\
\text { vs. } \\
\text { mitoxantrone + prednisone }\end{array}$ & mCRPC - post docetaxel & 755 & 15.1 vs. 12.7 \\
\hline COU-AA-301 & $\begin{array}{c}\text { abiraterone + prednisone } \\
\text { vs. } \\
\text { placebo + prednisone }\end{array}$ & mCRPC - post docetaxel & 1195 & 14.8 vs. 10.9 \\
\hline COU-AA-302 & $\begin{array}{c}\text { abiraterone + prednisone } \\
\text { vs. } \\
\text { placebo + prednisone }\end{array}$ & mCRPC - pre docetaxel & 1088 & NR vs. 27.2 \\
\hline AFFIRM & enzalutamide vs. placebo & mCRPC - post docetaxel & 1199 & 18.4 vs. 13.6 \\
\hline PREVAIL & enzalutamide vs. placebo & mCRPC - pre docetaxel & 1700 & 32.4 vs. 30.2 \\
\hline ALSYMPCA & alpharadin vs. placebo & $\begin{array}{l}\text { mCRPC - post docetaxel/not } \\
\text { qualified for docetaxel therapy }\end{array}$ & 921 & 14.9 vs. 11.3 \\
\hline
\end{tabular}


the FDA, mCRPC therapy is still combined with LHRH analogues. The basic argument supporting their use is a lack of studies which could confirm the effectiveness of the newest medications in monotherapy. It may be assumed that the recent drugs are not yet sufficiently effective to independently slow down the course of a metastatic disease, yet they present significant added value to LHRH analogue therapy. Important aspects which require further confirmation in clinical studies should also include the order of the mentioned therapies (sequential vs. simultaneous "cocktail"), and the possibilities of combining new medications.

The authors declare no conflict of interest. This article was supported by IPSEN (Warsaw, Poland).

\section{References}

1. Hammerer P, Madersbacher S. Landmarks in hormonal therapy for prostate cancer. BJU Int 2012; 110: 23-9.

2. Huggins C, Stevens RE Jr, Hodges CV. Studies on Prostatic Cancer: I Effect of Castration, of Estrogen and Androgen Injection on Serum Phosphatases In Metastatic Carcinoma of the Prostate. Arch Surg 1941; 43: 209.

3. Huggins C. Endocrine-induced regression of cancers. Cancer Res 1967; 27: 1925-30.

4. Page ST, Lin DW, Mostaghel EA, et al. Persistent intraprostatic an drogen concentrations after medical castration in healthy men. J Clin Endocrinol Metab 2006; 91: 3850-6.

5. Stanbrough M, Bubley GJ, Ross K, Golub TR, Rubin MA, Penning TM, Febbo PG, Balk SP. Increased expression of genes converting adrenal androgens to testosterone in androgen-independent prostate cancer. Cancer Res 2006; 66: 2815.

6. Holzbeierlein J, Lal P, LaTulippe E, et al. Gene expression analysis of human prostate carcinoma during hormonal therapy identifies androgen-responsive genes and mechanisms of therapy resis tance. Am J Pathol 2004; 164: 217-27.

7. Buchanan G, Irvine RA, Coetzee GA, Tilley WD. Contribution of the androgen receptor to prostate cancer predisposition and progression. Cancer Metastasis Rev 2001; 20: 207-23.

8. Mostaghel EA, Montgomery B, Nelson PS. Castration-resistant prostate cancer: targeting androgen metabolic pathways in recurrent disease. Urol Oncol 2009; 27: 251-7.

9. Taylor CD, Elson P, Trump DL. Importance of continued testicular suppression in hormone-refractory prostate cancer. J Clin Oncol 1993; 11: 2167-72.

10. Hussain M, Wolf M, Marshall E, Crawford ED, Eisenberger M. Effects of continued androgen-deprivation therapy and other prog nostic factors on response and survival in phase II chemotherapy trials for hormone-refractory prostate cancer: a Southwest Oncology Group report. J Clin Oncol 1994; 12: 1868-75.

11. Mounedji N, Lundstroem EA, Purcea P, Grosgurin P, Porchet HC. Ef ficacy of triptorelin in lowering serum testosterone (sT) in patients with advanced prostate cancer. J Clin Oncol 2011; 29; abstr: 162.

12. Perachino M, Cavalli V, Bravi F. Testosterone levels in patients with metastatic prostate cancer treated with luteinizing hormone-re leasing hormone therapy: prognostic significance? BJU Int 2010; 105: 648-51.

13. Berthold DR, Pond GR, Soban F, de Wit R, Eisenberger M, Tannock IF. Docetaxel plus prednisone or mitoxantrone plus prednisone for advanced prostate cancer: updated survival in the TAX 327 study. J Clin Oncol 2008; 26: 242-45.

14. Petrylak DP, Tangen CM, Hussain MH, et al. Docetaxel and estramustine compared with mitoxantrone and prednisone for ad vanced refractory prostate cancer. N Engl J Med 2004; 351: 1513-20.

15. de Bono JS, Oudard S, Ozguroglu M, et al. Prednisone plus cabazitaxel or mitoxantrone for metastatic castration-resistant pros tate cancer progressing after docetaxel treatment: a randomised open-label trial. Lancet 2010; 376: 1147-54.
16. de Bono JS, Logothetis CJ, Molina A, et al. COU-AA-301 Investigators. Abiraterone and increased survival in metastatic prostate cancer. N Engl J Med 2011; 364: 1995-2005.

17. Ryan CJ, Smith MR, de Bono JS, et al. COU-AA-302 Investigators. Abiraterone in metastatic prostate cancer without previous chemotherapy. N Engl J Med 2013; 368: 138-48.

18. Pinski J, Xiong S, Wang Q, Stanczyk F, Hawes D, Liu S. Effect of luteinizing hormone on the steroidogenic pathway in prostate cancer. Prostate 2011; 71: 892-8.

19. Mostaghel EA, Nelson P, Lange PH, et al. Neoadjuvant pathway suppression prior to prostatectomy. J Clin Oncol 2012; 30: abstr: 4520.

20. Taplin ME, Montgomery RB, Logothetis C, et al. Effect of neoadjuvant abiraterone acetate (AA) plus leuprolide acetate ( $\mathrm{LHRHa}$ ) on PSA, pathological complete response ( $P C R)$, and near $p C R$ in localized high-risk prostate cancer (LHRPC): results of a randomized phase II study. Proc Am Soc Clin Onc 2012; 30: abstr: 4521.

21. De Bono JS, Fizazi K, Saad F, et al. Primary, secondary, and quality-of-life endpoint results from the phase III AFFIRM study of MDV3100, an androgen receptor signaling inhibitor. J Clin Oncol 2012; 30: abstr. 4519.

22. Http://investors.medivation.com/releasedetail.cfm?ReleaseID $=798880$.

23. Costa L, Badia X, Chow E, Lipton A, Wardley A. Impact of skeletal complications on patients' quality of life, mobility, and functional independence. Support Care Cancer 2008; 16: 879-89.

24. Sabbatini P, Larson SM, Kremer A, et al. Prognostic significance of extent of disease in bone in patients with androgen-independent prostate cancer. J Clin Oncol 1999; 17: 948-57.

25. Ziółkowska E, Zarzycka M, Wiśniewski T, Żyromska A. The side effects of hormonal therapy at the patients with carcinoma of prostate cancer. Contemp Oncol 2012; 16 : 491-7.

26. Fizazi K, Carducci M, Smith M, et al. Denosumab versus zoledronic acid for treatment of bone metastases in men. Lancet 2011; 377: 813-22.

27. Parker C, Nilsson S, Heinrich D, et al. Alpha emitter radium-223 and survival in metastatic prostate cancer. N Engl J Med 2013; 369: 213-23.

28. Stawerski P, Wagrowska-Danilewicz M, Stasikowska-Kanicka O, Tuka E, Danilewicz M. Augmented mast cell infiltration and microvessel density in prostate cancer. Contemp Oncol 2013; 17: 378.

29. Kelly WK, Halabi S, Carducci M, et al. Randomized, double-blind, placebo-controlled phase III trial comparing docetaxel and prednisone with or without bevacizumab in men with metastatic castration-resistant prostate cancer: CALGB 90401. J Clin Oncol 2012; 1: $30: 1534-40$

30. Tannock IF, Fizazi K, Ivanov S, et al. Aflibercept versus placebo in combination with docetaxel and prednisone for treatment of men with metastatic castration-resistant prostate cancer (VENICE): a phase 3, double-blind randomised trial. Lancet Oncol 2013; 14: 760-8.

31. Armstrong AJ, Haggman M, Stadler WM, et al. Long-term survival and biomarker correlates of tasquinimod efficacy in a multicenter randomized study of men with minimally symptomatic metastatic castration-resistant prostate cancer. Clin Cancer Res 2013; 19: 6891-901.

\section{Address for correspondence}

\section{Tomasz Mileck}

Department and Clinic of Urology and Urologic Oncology

Poznan University of Medical Sciences

Szwajcarska 3

61-285 Poznan, Poland

e-mail: tmilecki@wp.pl

Submitted: 3.01.2014

Accepted: $\quad 26.03 .2014$ 